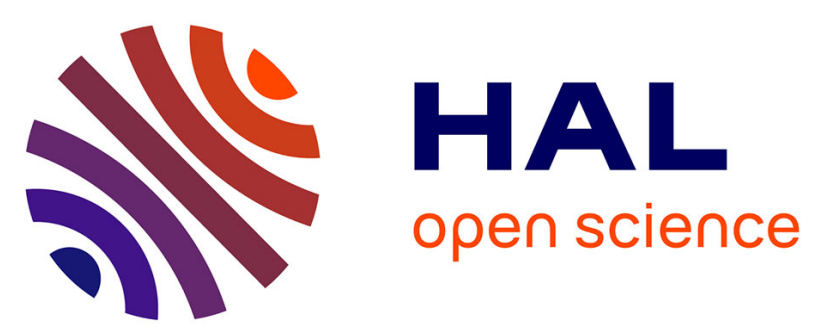

\title{
A parcel-based graph to match connectivity analysis with field action in agricultural landscapes: Is node removal a reliable method?
}

Jean-Christophe Foltête

\section{- To cite this version:}

Jean-Christophe Foltête. A parcel-based graph to match connectivity analysis with field action in agricultural landscapes: Is node removal a reliable method?. Landscape and Urban Planning, 2018, 178, pp.32-42. 10.1016/j.landurbplan.2018.05.016 . hal-01807332

\section{HAL Id: hal-01807332 \\ https://hal.science/hal-01807332}

Submitted on 4 Mar 2020

HAL is a multi-disciplinary open access archive for the deposit and dissemination of scientific research documents, whether they are published or not. The documents may come from teaching and research institutions in France or abroad, or from public or private research centers.
L'archive ouverte pluridisciplinaire HAL, est destinée au dépôt et à la diffusion de documents scientifiques de niveau recherche, publiés ou non, émanant des établissements d'enseignement et de recherche français ou étrangers, des laboratoires publics ou privés. 


\section{A PARCEL-BASED GRAPH TO MATCH CONNECTIVITY ANALYSIS WITH FIELD ACTION IN AGRICULTURAL LANDSCAPES: IS NODE REMOVAL A RELIABLE METHOD?}

Jean-Christophe Foltête*

ThéMA, UMR 6049 CNRS / University of Bourgogne Franche-Comté, 32, rue Mégevand F-25030 Besançon, France

* corresponding author

This is a postprint version, the definitive version of this paper is :

Foltête, J.-C. (2018). A parcel-based graph to match connectivity analysis with field action in agricultural landscapes : Is node removal a reliable method? Landscape and Urban Planning, 178, 32-42. https://doi.org/10.1016/j.landurbplan.2018.05.016

Highlights

- Field actions are planned to reduce functional connectivity of grassland rodents

- A parcel-based graph is constructed to prioritize locations for field actions

- Parcel rankings determined by removal method and land-use changes are compared

- Removal method and land-use changes yield similar rankings via enumerative approach

- The two methods differ when rankings are compared by a cumulative approach 
Patch-based graphs are widely used to display and quantify landscape connectivity. They are specially relevant for decision support in land planning and biological conservation. Matching connectivity analyses with practical actions in agricultural landscapes involves considering management units rather than habitat patches. However, at a local scale, the classical method for prioritizing graph elements (node removal) using connectivity indexes such as delta IIC could be viewed as a highly contrived approach with respect to the actual changes in land use. Here we address the relevance of this method compared to simulations likely to display these land-use changes in a more realistic way. Prioritization as determined by the removal method is tested here against simulated land-use changes in four scenarios (e.g. replacing grasslands by croplands) for an agricultural area in the Jura massif (eastern France) where field actions are undertaken to combat the spread of grassland rodents. The results obtained by ranking all the parcels ("enumerative" approach) show that the removal method provides rankings similar to those obtained with the land-use change scenarios, except for the planting of hedgerows. However, defining a limited number of key parcels ("cumulative" approach) results in different rankings whatever the scenarios. This shows that when applying parcelbased graphs to practical actions, the reliability of the removal method depends on the way the connectivity analysis is conducted. Simulating land-use changes, which is more realistic but more timeconsuming, proves relevant if only a few key parcels need to be identified for actions to be conducted in the field.

Keywords: agricultural parcel, landscape connectivity, connectivity metric, prioritization, landuse change. 


\section{Introduction}

The conservation of biodiversity involves preserving wildlife habitats and their accessibility, maintaining flows of individuals across landscapes, and ensuring population viability. Research dealing with this issue in landscape ecology and biological conservation has mainly focused on landscape connectivity, defined as the capacity of landscape to enable individuals to move across space (Taylor, Fahrig, \& With, 2006). Many studies of functional connectivity have been carried out for several decades now, either seeking to observe and better understand real flows in the field in order to characterize actual connectivity (Baguette, Blanchet, Legrand, Stevens, \& Turlure, 2013), or using modeling approaches to represent potential connectivity (Calabrese \& Fagan, 2004).

Among several methods capable of representing ecological networks and analyzing potential connectivity, landscape graphs are widely used because they offer a functional vision of these networks and do not require large amounts of ecological data, unlike individual-based models (Calabrese \& Fagan, 2004; Galpern, Manseau, \& Fall, 2011; Urban, Minor, Treml, \& Schick, 2009). As landscape graphs are spatially explicit models characterized by a very simple structure, they are suitable for providing decision support in conservation planning and landscape management. From this operational perspective, they can be used (1) to prioritize the most vulnerable elements (e.g. habitat patches) requiring protection so as to preserve the functioning of ecological networks; and (2) to identify the most relevant locations for action in the field so as to improve landscape connectivity (Foltête, Girardet, \& Clauzel, 2014). Many authors have outlined the efficiency of landscape graphs in addressing these operational issues. Some have focused on identifying locations for reforesting agricultural land (García-Feced, Saura, \& Elena-Rosseló, 2011), for creating and restoring ponds for amphibians (Clauzel, Bannwarth, \& Foltête, 2015), or for changing agricultural practices so as to stem the spread of rodent species (Foltête, Couval, Fontanier, Vuidel, \& Giraudoux, 2016). Other studies have addressed the design of wildlife corridors (Zetterberg, Mörtberg, \& Balfors, 2010; Loro, Ortega, Arce, \& Geneletti, 2015) or wildlife crossings along linear infrastructures (Gurrutxaga \& Saura, 2013; Mimet, Clauzel, \& Foltête, 2016; Girardet, Foltête, Clauzel, \& Vuidel, 2016).

One strong point of graph-based methods applied to landscape is that they capture a complex array of elements and relationships within a simple structure composed of two sets of objects: nodes representing habitat patches and links representing functional distances between patches. This simple structure can be readily used in numerous procedures such as computing connectivity metrics and simulating changes in the network. As a result, these procedures provide outcomes that can be translated in terms of decision support in response to operational issues (Foltête, Girardet, \& Clauzel, 2014). One of the most popular procedures is the removal method consisting in simulating the removal of each node successively and quantifying the impact of such modifications on connectivity, with the aim of identifying the key patches. The removal method was first applied to landscape graphs by Keitt, Urban, \& Milne (1997) and is the basis for computing the PC index (Saura \& Pascual-Hortal, 2007) and IIC index (Pascual-Hortal \& Saura, 2006), two widely used network-level metrics. Beyond identifying key patches for prioritizing conservation measures, the search for the best locations for carrying out field actions such as landscaping to increase (or sometimes decrease) connectivity can be based on the same principle (Foltête, Girardet, \& Clauzel, 2014). 
The method consisting in simulating patch removal in landscape graphs is a relevant way of investigating operational issues. However, in practice, the use of this method for taking concrete action in the field is facilitated when the elements to be analyzed (i.e. the nodes of the graph) correspond to management units belonging to private or public landowners. Such a situation could perhaps be encountered in the case of specific habitat patches characterized by their small size and their being "naturally" fragmented, for example a set of ponds. But in many cases, such correspondence is impossible because habitat patches usually considered as the nodes are defined on the basis of ecological criteria alone. In a recent study applied to an agricultural landscape, the difficulty in making a patch-based graph operational arose from the mismatch between the large size of the grassland patches involved in the connectivity analysis and the small size of the parcels managed by farmers on which actual actions could be conducted (Foltête, Couval, Fontanier, Vuidel, \& Giraudoux, 2016). Such a situation can be illustrated by a fictitious example in which both options (patches vs parcels) are compared (Fig. 1). This leads us to consider connectivity graphs based on spatial units defined by management criteria and to question their relevance for operational issues.

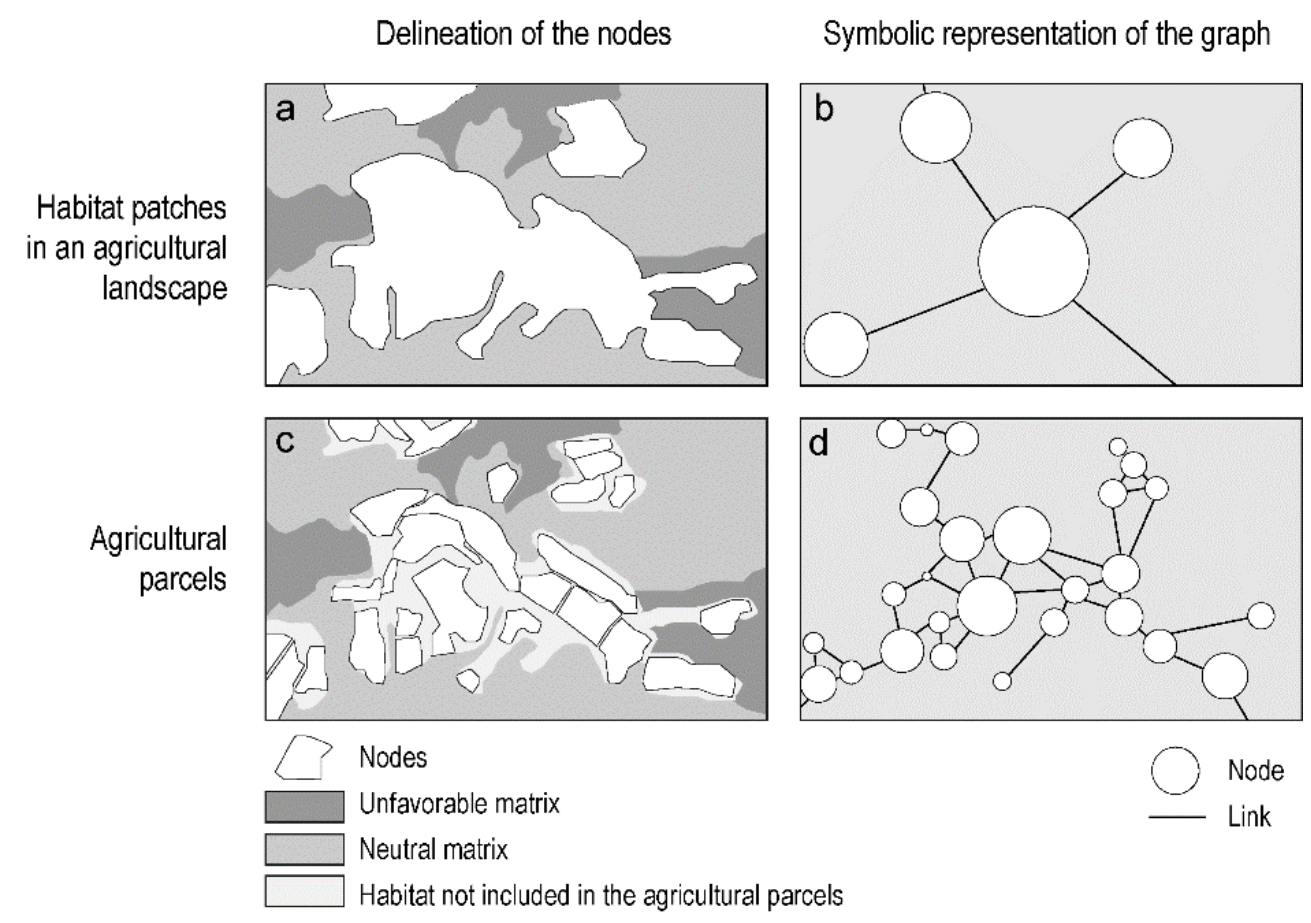

Figure 1 : Patches vs parcels, two ways of considering nodes in agricultural landscapes. The classical method for mapping nodes consists in delineating areas corresponding to the preferential habitat of the species under study (e.g., grassland) (a). An alternative is to consider agricultural parcels to be the nodes (b). In this case, the parcels within a given patch may be adjacent or separated by a portion of habitat not included in the parcels. The decision support provided by connectivity analyses differs according to these options. In the case of patch-based graphs (c), the removal method will lead to field actions being contemplated on large zones including several parcels and other areas. This may raise practical problems (e.g., do all the parcel managers agree with changes in their respective parcels?). With parcel-based graphs (d), the connectivity analysis is directly geared to the potential actions in the field and can be applied to the subset of parcels of which the managers are involved.

In the case of agricultural landscapes, using management units for conducting connectivity analyses entails building parcel-based graphs instead of patch-based graphs. This assumes that the spatial grain of the analysis is fine enough to include the parcels in the land-use map. In this way, the 
results of connectivity analyses are expected to be more readily convertible into concrete actions in the field. However, in this case, the removal method may involve overkill with respect to the real situation, because a parcel cannot be simply removed. In the real world, a node (patch or parcel) that is removed is replaced by some other land use and, if this change modifies the resistance of the matrix and also modifies the local topology of the graph, its impact may differ from changes quantified by removal alone. Consequently, the binary mode (presence/absence) of the removal method is better adapted to contexts where the landscape matrix is assumed to be uniform (i.e. when the links are weighted by Euclidean distances), than to cases of heterogeneous matrixes, i.e. when using least-cost or resistance distances. Furthermore, in some cases, the actual changes in land use may affect only a part of the node area, for example if hedgerows are planted in open-habitat nodes. In this case, the nodes are not removed but their quality is altered. For all these reasons, prioritizing actions on the basis of the removal method could prove irrelevant when it comes to properly representing changes in agricultural practices that may correspond to more subtle land-use modifications.

When it comes to transposing the graph-based connectivity analyses from patches to parcels (i.e. to apply these analyses at the scale of the management units), the question is therefore whether the removal method provides reliable results for prioritization compared to simulations of more realistic changes in agricultural parcels. This question has to be addressed in order to detect any potential contradiction between the spatial scale suitable for concrete actions in the field and the prioritization method that could be too coarse for simulating land-use changes.

In this paper, we propose to investigate the relevance of a parcel-based graph in addressing an issue of reduction of connectivity in a grassland network. In the Jura massif (eastern France), the cyclic spread of the montane water vole (Arvicola terrestris sherman) causes numerous ecological, economic, and public health problems. Populations of montane water vole (Arvicola terrestris sherman) spread in grasslands in about cyclic outbreaks over about five decades (Blant, Beuret, Poitry, \& Joseph, 2009; Delattre \& Giraudoux, 2009). The main determinants of the diffusion of these populations have been studied by Duhamel, Quéré, Delattre, \& Giraudoux (2000), Morilhat, Bernard, Foltête, \& Giraudoux (2008), and Berthier et al. (2014). These studies have shown that homogeneous grassland openfields intensify and accelerate the spread of the montane water vole, unlike mosaics mixing grasslands, hedgerows, and wooded patches. Knowing that anticoagulant rodenticides such as bromadiolone also kill non-target wildlife species and prove ineffective in the long run (Coeurdassier et al., 2014), a major issue is to define agro-ecological interventions to limit the connectivity of grasslands. In this context, the purpose is to use a landscape graph to identify the key areas of the grassland network likely to be the most appropriate areas for carrying out field actions to limit grassland connectivity.

At the regional scale, several studies have shown that a graph based on grassland patches provides a suitable framework for representing the regional-level ecological network of the montane water vole (Foltête \& Giraudoux, 2012) and conducting connectivity analyses (Foltête et al., 2016; Foltête \& Vuidel, 2017). Here we focus instead on a small area where agricultural changes and specific field actions may be contemplated to limit connectivity of the species. Using a modeling approach, several scenarios are compared to the "classical" removal method: replacing grasslands by cereal crops, planting hedgerows, setting up perches for birds of prey, and combining both hedgerows and perches. Given the relative stability of the patch ranking derived from the removal method observed in previous studies (Avon \& Bergès, 2016; Blazquez-Cabrera, Bodin, \& Saura, 2014), our hypothesis is that the removal method provides similar rankings to simulations of land-use changes and 
consequently indicates reliable outcomes although it does not always represent realistic changes. The present study aims at verifying this hypothesis and clarifying the possible differences between the removal method and the simulation of land-use changes corresponding to the scenarios indicated.

\section{Material and methods}

\subsection{Study area and spatial data}

The study area extends over $74 \mathrm{~km} 2$ of the eastern part of the Jura massif, on the border between France and Switzerland. It consists of a plateau rising from $700 \mathrm{~m}$ to $850 \mathrm{~m}$, covered by a mosaic of grasslands, hedgerows, and wooded patches. This plateau is surrounded by the deep and forested valley of the river Doubs that here forms the border between France and Switzerland (Fig. 2). A land-use map was built by combining several data sources. The BD Topo IGN 2010 database was used to map buildings, the hydrographic network, transport infrastructures, and forests (deciduous, evergreen, and mixed). The 2010 "Registre Parcellaire Graphique" farming database was used to classify agricultural areas into two categories: annual crops and grasslands. Two steps were then added in order to map hedgerows. Wide hedgerows (at least $25 \mathrm{~m}$ wide) were extracted from the layer describing forests by applying a morphological analysis (Vogt et al., 2007). Narrow hedgerows (10-25 $\mathrm{m}$ wide and at least $25 \mathrm{~m}$ long) were identified from aerial photos with a spatial resolution of $0.5 \mathrm{~m}$. The two types of hedgerows were then aggregated into a single class. The areas unclassified after compiling all these data were identified by photo-interpretation as herbaceous areas. A map containing nine land-cover classes was finally produced in raster format with a spatial resolution of 10 m (Fig. 2).

The boundaries of grassland parcels were extracted from the 2010 "Registre Parcellaire Graphique" database. It should be noted that these spatial units are not real cadastral parcels but "islets" of parcels managed by the same farmer, registered and delineated for the Common Agricultural Policy of European Union. However, we will use the term "parcel" in what follows. To distinguish the parcels as objects when superimposed on the land-cover map and to represent fenced areas and areas of wild grasses, their boundaries were assigned to the "other herbaceous areas" class. 


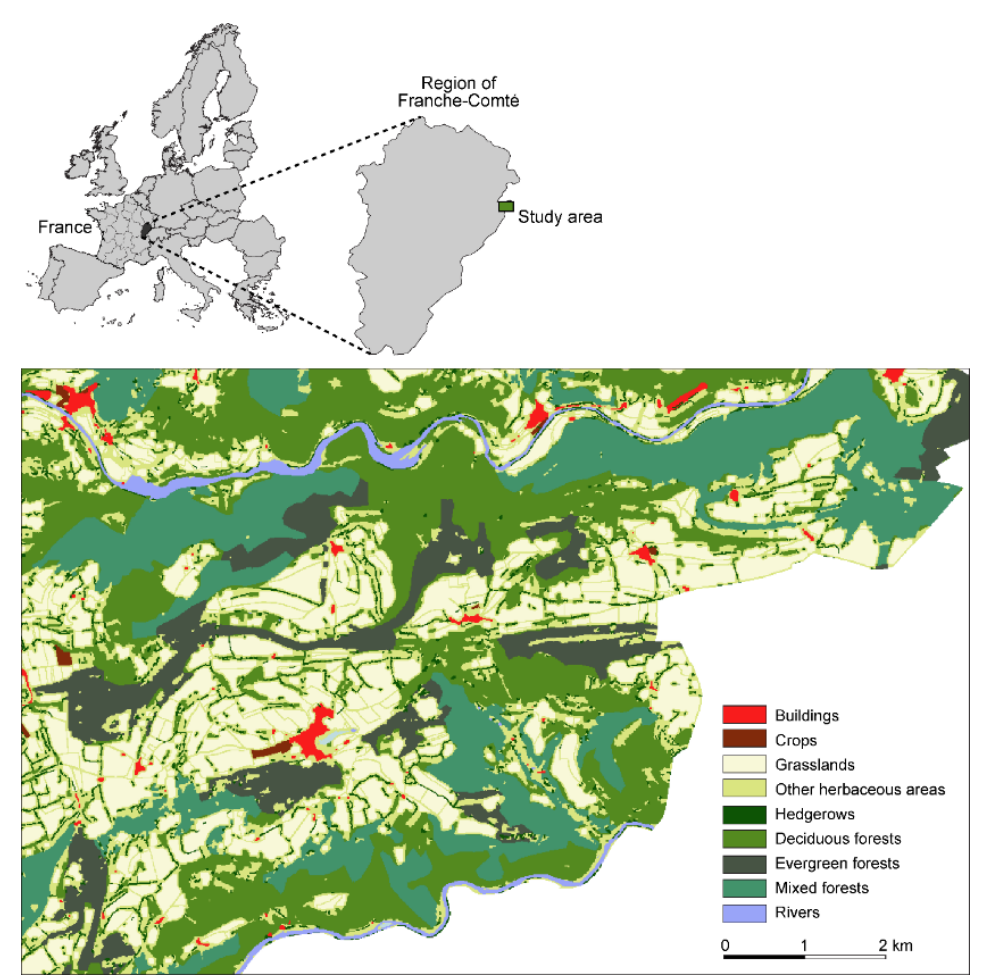

Figure 2 : Land-use map in the study area

\subsection{Connectivity analysis}

A minimal planar graph, i.e. a graph with a planar topology defined from Voronoi polygons and without link thresholding (Fall, Fortin, Manseau, \& O'Brien, 2007), was designed to represent the grassland network. The grassland parcels were taken to be the nodes of this graph. The links were weighted by the least-cost distance between parcels, computed from the set of resistance values calibrated in Foltête \& Giraudoux (2012) and used in Foltête et al. (2016) and Foltête \& Vuidel (2017). These resistance values are presented in Appendix 1.

The set of nodes and links was considered as the initial graph for applying the usual removal method. For this purpose, the probability of connectivity (PC) (Saura \& Pascual, 2007) was chosen to be the metric of reference, allowing connectivity to be quantified by including both the amount of habitat and its degree of connectivity such that:

$$
P C=\frac{1}{A^{2}} \sum_{i=1}^{n} \sum_{j=1}^{n} a_{i} a_{j} p_{i j}^{*}
$$

where $p_{i j}^{*}$ is the maximum probability of movement between the parcels $i$ and $j, a_{i}$ and $a_{j}$ are the areas of the parcels $i$ and $j, A$ is the total area of the study zone, and $n$ is the number of parcels. The probability $p_{i j}$ is obtained by transforming the distance $d_{i j}$ between parcels $i$ and $j$ by an exponential function such that:

$$
p_{i j}=e^{-\alpha d_{i j}}
$$

where $\alpha$ represents the decrease in the probability of movement with distance. The value of the parameter $\alpha$ was set so that a distance of 1000 cost units (approximately $4 \mathrm{~km}$ ) corresponded to a probability of movement of 0.5 . The correspondence between 1000 cost units and $4 \mathrm{~km}$ was 
established from a regression applied to the set of links, where the logarithm of cost distances was considered as a linear function of the logarithm of metric distances (see Foltête \& Giraudoux, 2012). This setting was identified as the most relevant for representing grassland networks of the montane water vole in Foltête \& Giraudoux (2012).

Given all these settings, the removal method was applied to the parcels successively so as to compute the $d P C$ for each parcel (Saura \& Pascual, 2007). This local indicator is defined as $A P C=$ $\left(P C-P C^{\prime}\right) / P C$ where $P C$ is the initial value when all parcels are present and $P C^{\prime}$ is the value of the metric when a given parcel is removed. The resulting values of $d P C$ were ranked to identify the parcels where a land-use change may have the biggest impact on montane water vole connectivity. The same approach was adopted by considering the three fractions of the $d P C$ proposed by Saura \& Rubio (2010), each representing a specific contribution to the calculation of $d P C$ : area of the parcels $\left(d P C_{\text {intra }}\right)$, potential dispersal fluxes towards surrounding parcels $\left(d P C_{\text {flux }}\right)$, and transit linking other parcels ( $d P C_{\text {connector }}$.

\subsection{Scenarios of landscaping and agricultural changes}

Four scenarios were defined to represent the potential changes in a more realistic manner than by parcel removal (Fig. 3). All these changes in land use have already been experimented on the Jura massif, e.g. Foltête et al. (2016). The first scenario is to convert the grassland parcels into cereal crop parcels, because the plowing required before planting cereal crops destroys vole galleries (Bonnet et al., 2013; Jacob \& Hempel, 2003; Jug, Brmez, Ivezic, Stipesevic, \& Stosic et al., 2008). The second scenario consists in adding hedgerows to all the edges of a given parcel, which is liable to increase the accessibility of avian and mammal predators to vole populations. At the landscape level, the role of dense hedgerow networks is indeed expected to have a negative effect on the abundance of small mammals (Michel, Burel, \& Butet, 2006). The third scenario is to erect artificial perches for birds of prey (e.g. buzzards) (Kay, Twigg, Nicol, \& Korn, 1994; Andersson, Wallander, \& Isaksson, 2009). Finally, the fourth scenario is to combine hedgerows and perches so as to favor predation by birds and mammals alike. As it applied to the entire area of the parcels, the first scenario is expected to have a greater impact and to provide results similar to node removal. The others scenarios do not involve the total removal of areas of grassland and so are expected to yield more specific results (Fig. 3). 
a
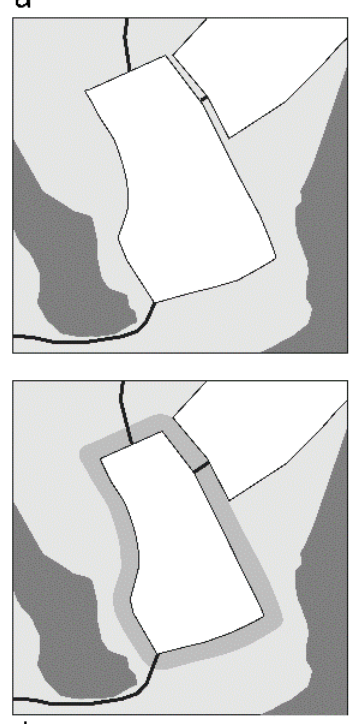

d

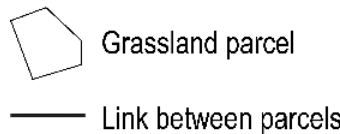

b
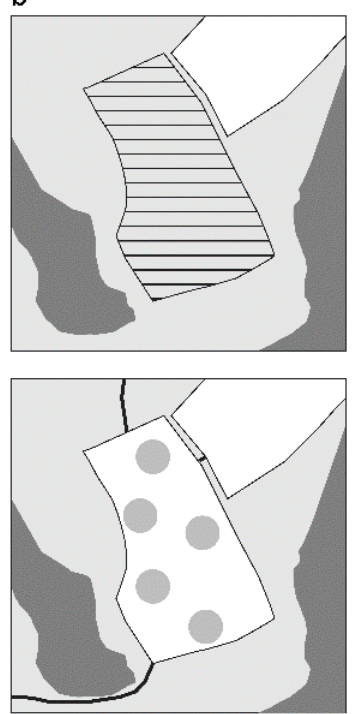

e

Deciduous forests

Crops, hedgerows or areas around perches

Other herbaceous areas

No data
C
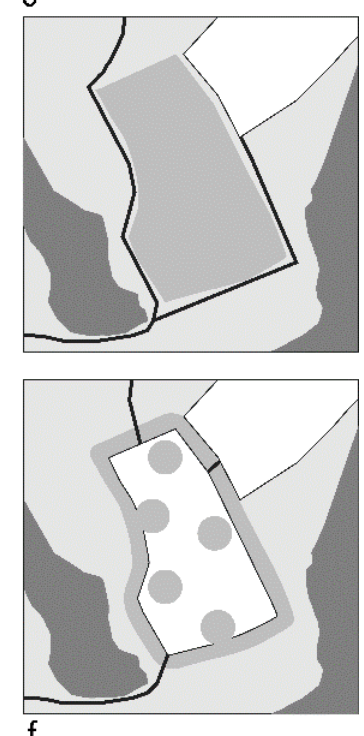

f

Figure 3 : Representation of scenarios for a fictitious parcel. From the current state (a) changes affecting the central parcel are simulated by node removal and four scenarios. Node removal renders the area of the focal parcel "inactive" and also implies removal of the links connected to this parcel (b). The planting of cereal crops produces a relative barrier affecting the new least-cost paths (c). The planting of hedgerows does not influence the graph topology but decreases the area of the parcel while increasing the cost of the links (d). Installing perches merely decreases the area of the parcel (e). Lastly the combination of hedgerows and perches is of the same type as the previous scenarios but with a stronger effect on the parcel area (f).

While the usual removal method is based on a single graph built from the initial land-cover map, the simulation of the previous scenarios involves applying a specific procedure for each parcel in turn. As any land-use change may induce a change in the topological structure of the graph (addition or removal of a link), this procedure consists in locally modifying the land-cover map, updating the graph, and finally computing connectivity values. This time-consuming procedure already used to investigate the past changes in land use (Sahraoui, Foltête, \& Clauzel, 2017) was implemented with Graphab 2.0. software (Foltête, Clauzel \& Vuidel, 2012). For a given scenario and a given parcel, the software automatically simulates the land-use changes to be made, designs the new graph, and computes the new $P C$ value. The set of these new values led us to define four alternative metrics in a similar way to the $d P C$ but based on the scenarios presented above: $d P C_{\text {crop }}, d P C_{\text {hedgerow, }} d P C_{\text {perch, }}$ and $d P C_{\mathrm{hp}}$ (hp standing for hedgerows and perches combined).

Each type of field action in the parcels was represented in a specific manner on the land-cover map. For a given parcel, the conversion of grassland into cropland was represented simply by a change in cost ( 25 instead of 1 ) for all the relevant pixels. The simulated hedgerows were defined by an internal buffer set to 10 meters (i.e. 1 pixel) wide. The perches were figured by 20 m-radius discs with a cost value of 25 , representing high predation areas around the focal points. A regular grid could have been used for locating these points, but some of the smaller parcels would then have had no potential perches. We thus chose to randomly define these locations; to do this, a large number of potential locations for artificial perches (5000) was initially defined in grasslands and then, by removing all 
perches located within $100 \mathrm{~m}$ of another perch, 3605 perches were selected for the entire study area. The minimum $100 \mathrm{~m}$ spacing was chosen following Kay et al. (1994).

\subsection{Two ways of implementing the scenarios}

With the aim of minimizing connectivity, the question is now to compare the results of prioritization obtained by the removal method and under each scenario. Two approaches can be used:

(1) An enumerative procedure where each potential change to a parcel is processed separately, as with the calculation of the $d P C$ and its three fractions. For a given scenario and for $n$ parcels, this procedure involves computing the reference connectivity metric $n$ times.

(2) A cumulative process consisting in validating at each step the parcel in which the land-use change makes the biggest impact, before finding the "next best" parcel, up to an initially defined total number of parcels. In this case, the global metric $P C$ was considered as the criterion to minimize at each step. This stepwise procedure was described in Foltête et al. (2014) and applied in Clauzel et al. (2015). It is expected to provide a more relevant combination of parcels because a simple enumeration may lead to the inclusion of parcels that are redundant for minimizing connectivity. However, it should be noted that the step-by-step procedure is more time-consuming than the enumeration procedure because testing $n$ parcels involves computing the reference metric $n^{2}$ times (in the present case 469225).

Both approaches were applied in this study. The enumerative procedure was performed on all the parcels whereas the cumulative procedure was limited to the best 20 parcels.

\subsection{Statistical analysis}

Under the enumerative procedure, the overview of the relationships between all the connectivity metrics was provided by a principal component analysis (PCA). Since the useful criterion for applying $d P C$ metrics in a decision support perspective is the ranking of the values, this PCA was carried out from the Spearman correlation coefficient matrix.

To be consistent with the operational goal of our study, the analyses were then focused on a small selection of parcels. The decision-making processes thus consist in selecting only the key parcels in terms of connectivity, i.e. the number of parcels being limited by the cost (or other constraint) of field actions such as landscaping or changing agricultural practices. From this perspective, we investigated the similarity between the selection of the key parcels resulting from the removal method ( $d P C$ and its three fractions) and the same process applied under the four scenarios. For a given number of key parcels $k$ (here $k=20$ ), we used a percentage of similarity defined as the proportion of common parcels between two sets of parcels selected from the first to the $k^{\text {th }}$. This means that a high percentage of similarity could be attained if the parcels were included in the same group of ranks, even if the rank of each parcel differs between the two sets.

The ranking differences between the removal method and the land-use changes were synthesized by counting the number of times the parcels were selected by each method among the first 20 parcels. The results of this counting allowed us to arrange the 685 parcels into five categories:

- All: parcels selected both by the removal method and at least two of the four land-use scenarios.

- Removal: parcels selected by the removal method only. 
- Land use: parcels selected by at least two of the four land-use scenarios but not selected by the removal method.

- Specific land use: parcels selected by a single land-use scenario and not selected by the removal method.

- Not selected: parcels never selected.

The four categories of the selected parcels were compared in terms of area using an ANOVA. This analysis is designed to separate the variability of the areas between the categories and within the categories, and to evaluate the significance of this difference by performing a Fisher test (Ott \& Longnecker, 2015).

\subsection{Computation}

The graphs were constructed, connectivity metrics computed, and land-use changes simulated using Graphab 2.0 (Foltête et al., 2012, see http://thema.univ-fcomte.fr/productions/graphab/).

\section{Results}

In the study area, 685 grassland parcels and 1891 links were identified to draw the initial graph, providing a $P C$ index of 0.008951 .

\subsection{Application of the enumeration procedure}

The 20 highest values of $d P C$ metrics were first investigated without taking into account the selected parcels (Fig. 4). Two groups of curves are clearly separated: $d P C, d P C_{\text {flux }}$, and $d P C_{\text {crop }}$ have values above 0.02 while the others are invariably below 0.01 , except for the first removal for

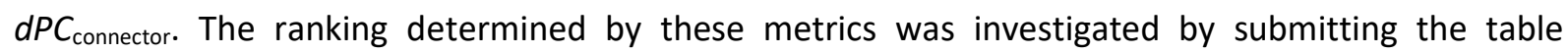
containing the 685 parcels to a PCA. In the correlation circle of the first two factors (Fig. 5), all metrics are located on the right-hand side of the circle (i.e. positively correlated with the first factor), meaning they globally provide similar ranks between parcels. Some of them differ from the others with respect to the second factor specifically opposing $d P C_{\text {connector }}$ and $d P C_{\text {hedgerow }}$ in positive coordinates and $d P C_{\text {intra }}$ in negative coordinates. However, this difference is not great since the absolute values of their coordinates are small. 


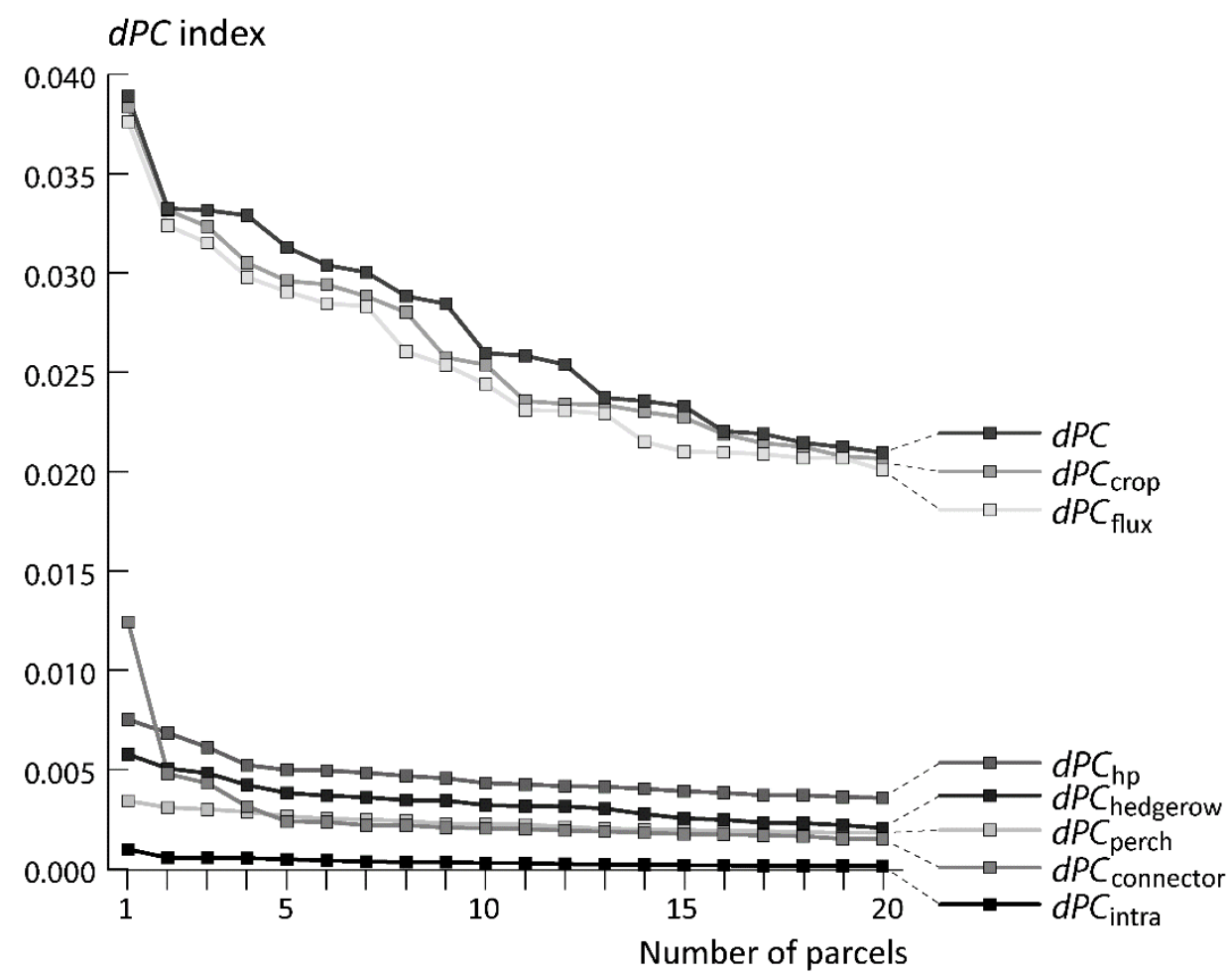

Figure 4 : Comparison of the 20 highest values of the dPC metrics

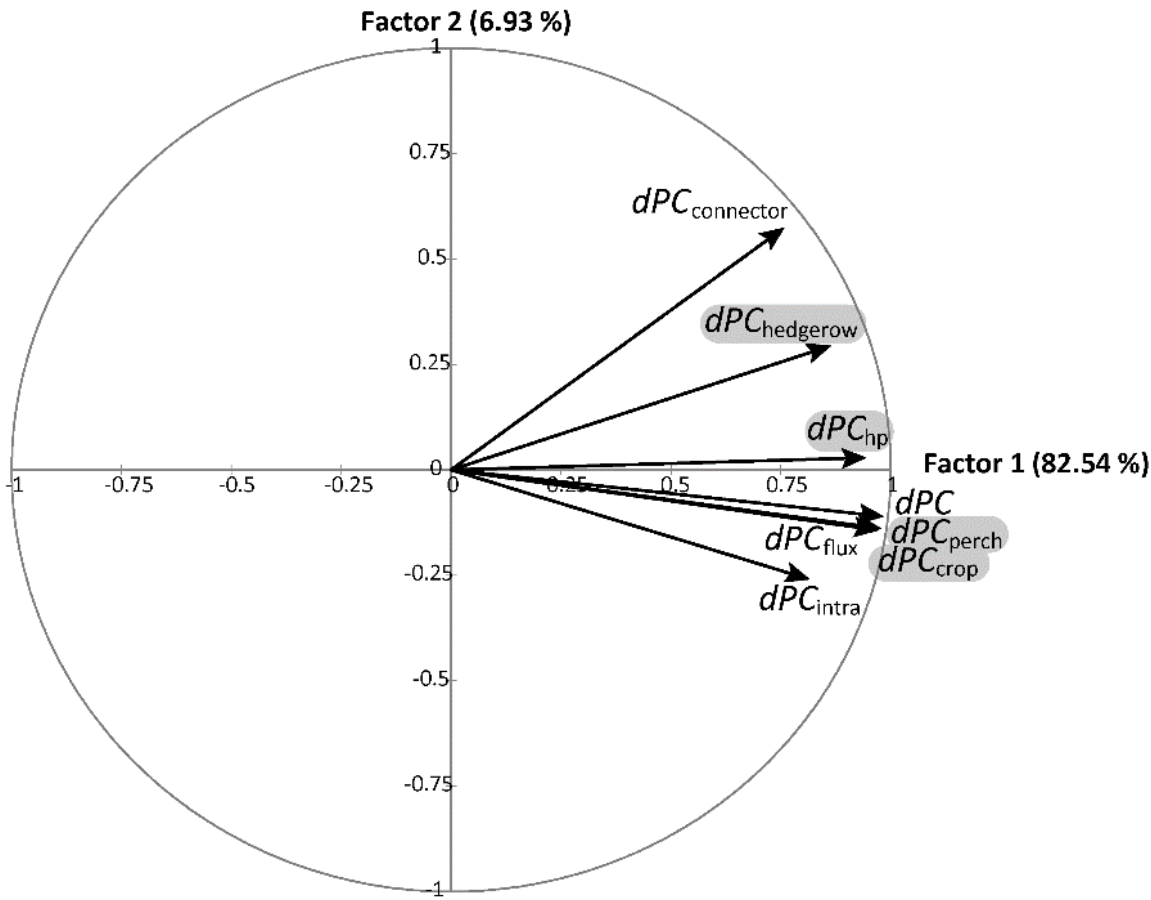

Figure 5: Principal component analysis correlation circle applied to the connectivity metrics. The characters highlighted in grey indicate the metrics computed from land-use change scenarios.

We then focused on the key parcels identified by the eight metrics. The percentage of similarity of the rankings was determined for the first 5, 10, and 20 parcels in each case (Table 1). Globally, the 
parcels selected by ranking the $\mathrm{dPC}$ computed from each land-use change scenario often differ from those selected using the classical dPC, however many key parcels there are (first part of Table 1). However, the degree of similarity is variable, this difference being more marked for dPChedgerow (maximum of $20 \%$ ) and dPChp (maximum of $50 \%$ ) than for dPCcrop and dPCperch giving at least $80 \%$ of the same parcels. The ranking based on the dPCintra provides analogous results but with lower degrees of similarity on the whole. The degrees of similarity obtained from dPCflux are the most contrasted, reaching at least $95 \%$ with $\mathrm{dPC}$ crop and $80 \%$ with dPCperch, but being conversely very low (between 0 and 20\%) with dPChedgerow and moderate with dPChp. Finally the ranking based on $\mathrm{dPC}$ connector proves to be least able to provide results similar to the four land-use change scenarios, even if the degrees of similarity with dPChedgerow and dPChp are around $50 \%$.

\begin{tabular}{|c|c|c|c|c|c|}
\hline Classical & Number & & Land-use c & cenarios & \\
\hline metrics & of key parcels & $d P C_{\text {crop }}$ & $d P C_{\text {hedgerow }}$ & $d P C_{\text {perch }}$ & $d P C_{\mathrm{hp}}$ \\
\hline$d P C$ & 5 & 80 & 20 & 80 & 40 \\
\hline & 10 & 80 & 10 & 80 & 50 \\
\hline & 20 & 95 & 10 & 90 & 50 \\
\hline$d P C_{\text {intra }}$ & 5 & 60 & 0 & 80 & 0 \\
\hline & 10 & 90 & 0 & 80 & 30 \\
\hline & 20 & 80 & 15 & 75 & 40 \\
\hline$d P C_{\text {flux }}$ & 5 & 100 & 0 & 80 & 20 \\
\hline & 10 & 100 & 0 & 90 & 40 \\
\hline & 20 & 95 & 20 & 90 & 50 \\
\hline$d P C_{\text {connector }}$ & 5 & 0 & 60 & 0 & 60 \\
\hline & 10 & 10 & 60 & 10 & 50 \\
\hline & 20 & 15 & 55 & 15 & 50 \\
\hline
\end{tabular}

Tableau 1 : Percentage similarity between the selection of key parcels by the classical removal method ( $d P C$ and its three fractions) and under each land-use change scenario. These percentages are computed by counting the number of common parcels among the first ranks $(5,10$, and 20 respectively).

The map of the results of the removal methods based on the $d P C$ metric and those obtained under the hedgerow-planting scenario show how the differences in ranking may be reflected in geographical space (Fig. 6). In each graph, the parcels characterized by the higher $d P C$ (respectively $d P C_{\text {hedgerow}}$ ) values are numbered from 1 to 20 . All these parcels are located in the central part of the 
area (corresponding to the main plateau of this zone), but in the first case (a) the key parcels are evenly distributed from west to east whereas in the second case (b) the key parcels are concentrated in specific areas. Among the largest parcels, only the second one is common to both cases, which is in keeping with what was observed from Table 1 about a degree of similarity of only $10 \%$.
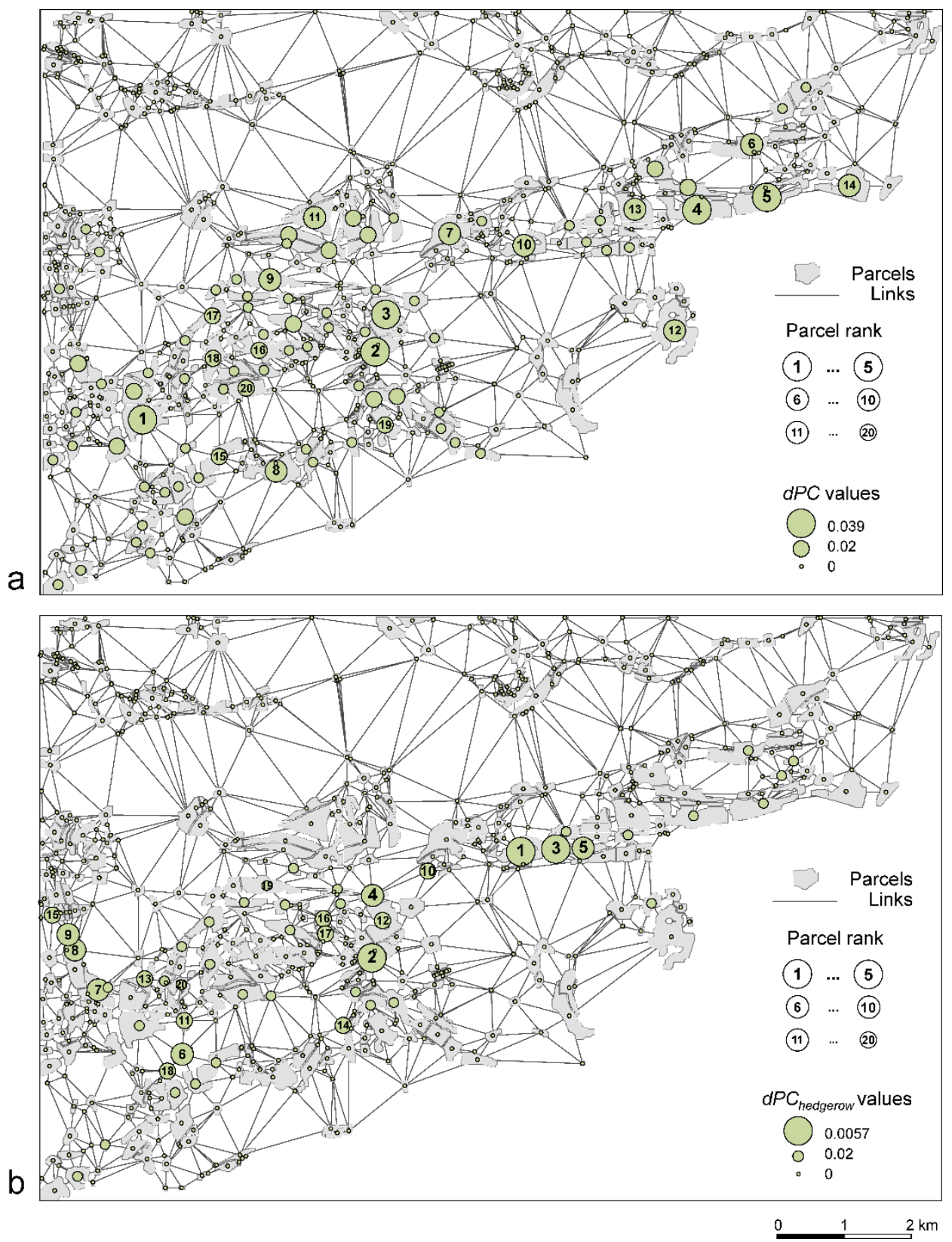

Figure 6 : Prioritization of parcels by (a) the node removal method and (b) hedgerow planting simulation. The node removal method involved computing the $A P C$ metric. The planting of virtual hedgerows entailed calculating the $d P C_{\text {hedgerow }}$ metric. In each case, the values were ranked so as to identify the 5, 10, and 20 key parcels where field action is most likely to limit connectivity of the montane water vole.

\subsection{Application of the cumulative procedure}

Starting from the same initial graph, the cumulative procedure was applied to minimize the $P C$ index until 20 parcels were removed. The same procedure was carried out for the four land-use change 
scenarios. The $P C$ index curves decrease as the parcels are removed for the five cases investigated, ignoring parcel ranks (Fig. 7). The curve resulting from parcel removal lies below all the others, although the curve for the cereal crop scenario is of a similar shape, and both attain a $P C$ index value of less than 0.005 . Conversely, the perch, hedgerow, and combined hedgerow and perch scenarios all provide comparable curves with shallower slopes and with a $P C$ index remaining above 0.008 .

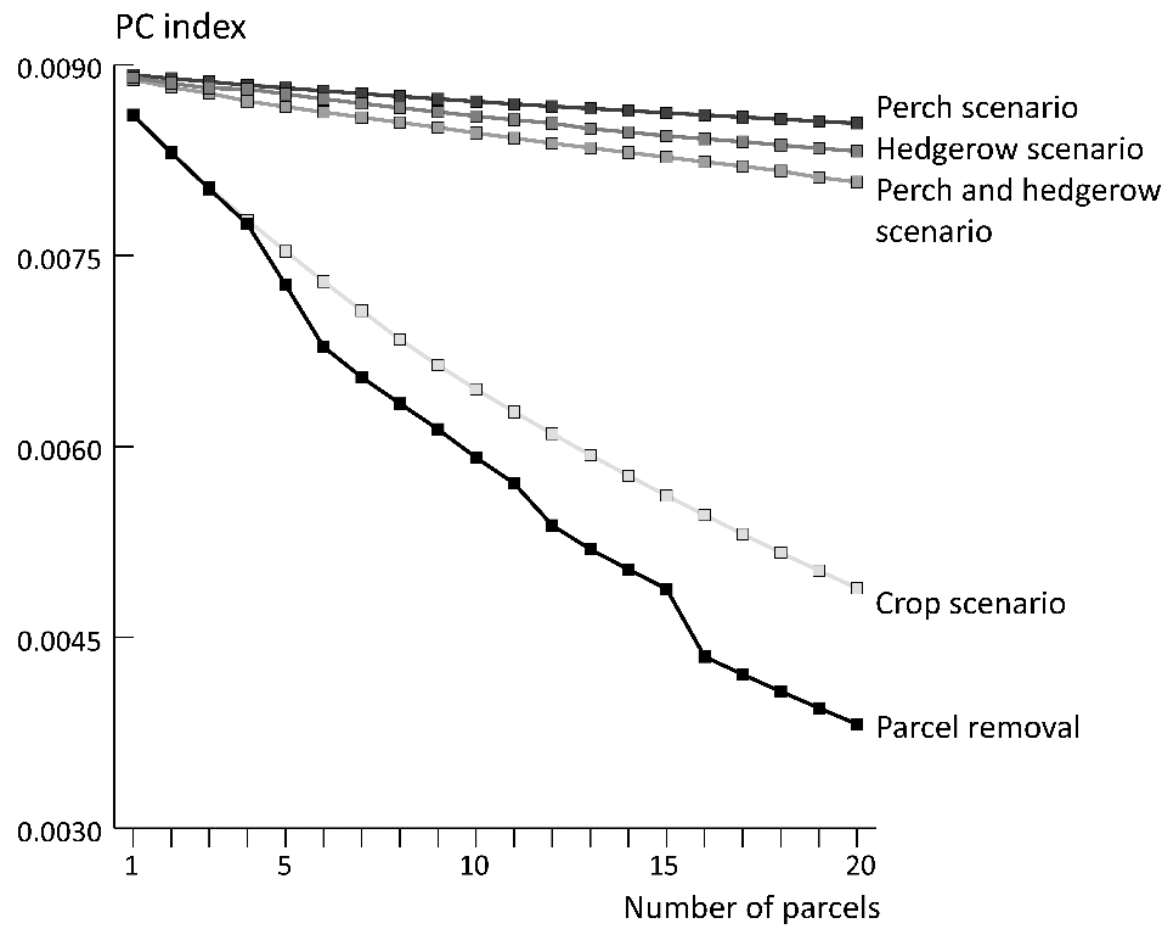

Figure 7 : Connectivity level (PC index) according to the number of parcels considered. The black curve represents the parcel removal method. The other curves represent land-use change scenarios.

As previously, the results of prioritization provided by the removal method and by the four scenarios were compared in terms of ranking similarity for 5, 10, and 20 parcels (Table 2). Except for the first five parcels defined from the hp scenario ( $80 \%$, i.e. $4 / 5$ parcels in common), the removal method does not reach a high degree of similarity, remaining around $60 \%$ in most cases. Here again, the hedgerow planting scenario proves to be the most specific with only $15 \%$ of common parcels among the top 20 parcels selected.

\begin{tabular}{|r|r|r|r|r|}
\hline \multirow{2}{*}{ Numbers } & \multicolumn{4}{|c|}{ Land-use change scenarios } \\
\cline { 2 - 5 } of key parcels & $d P C_{\text {crop }}$ & $d P C_{\text {hedgerow }}$ & $d P C_{\text {perch }}$ & $d P C_{\mathrm{hp}}$ \\
\cline { 2 - 5 } & 60 & 40 & 60 & 80 \\
10 & 60 & 20 & 60 & 60 \\
20 & 65 & 15 & 65 & 55 \\
\hline
\end{tabular}

Tableau 2 : Percentage similarity between the selection of key parcels by the removal method and under each landuse change scenario. These percentages are computed by counting the number of common parcels among the first ranks $(5$, 10 , and 20 respectively). 
The ranking differences between the removal method and the land-use changes were synthesized by counting the number of times the parcels were selected by each method among the first 20 parcels. The results of this counting allowed us to arrange the 685 parcels into five categories:

- All: parcels selected both by the removal method and at least two of the four land-use scenarios (15 units).

- Removal: parcels selected by the removal method only ( 5 units).

- Land use: parcels selected by at least two of the four land-use scenarios but not selected by the removal method (10 units).

- Specific land use: parcels selected by a single land-use scenario and not selected by the removal method (14 units).

- Not selected: parcels never selected (641 units).

On the map of this classification, the four categories of selected parcels are distributed across the entire area except for its northern part (Fig. 8). Beyond this apparently fortuitous event, the removal category seems mainly to concern the central core of the network. The parcels in the all category appear to be larger than others on average. Those of the land use category seem quite large too compared to the removal and specific land use categories. Conversely the parcels selected only under a specific land use scenario are small. Their selection from among the 20 key parcels cannot be easily explained and would require a case-by-case investigation. Although the number of selected parcels (44) is not enough to conduct a rigourous statistical analysis, the difference in parcel area between the categories was considered significant by an ANOVA $(p<0.0001)$.

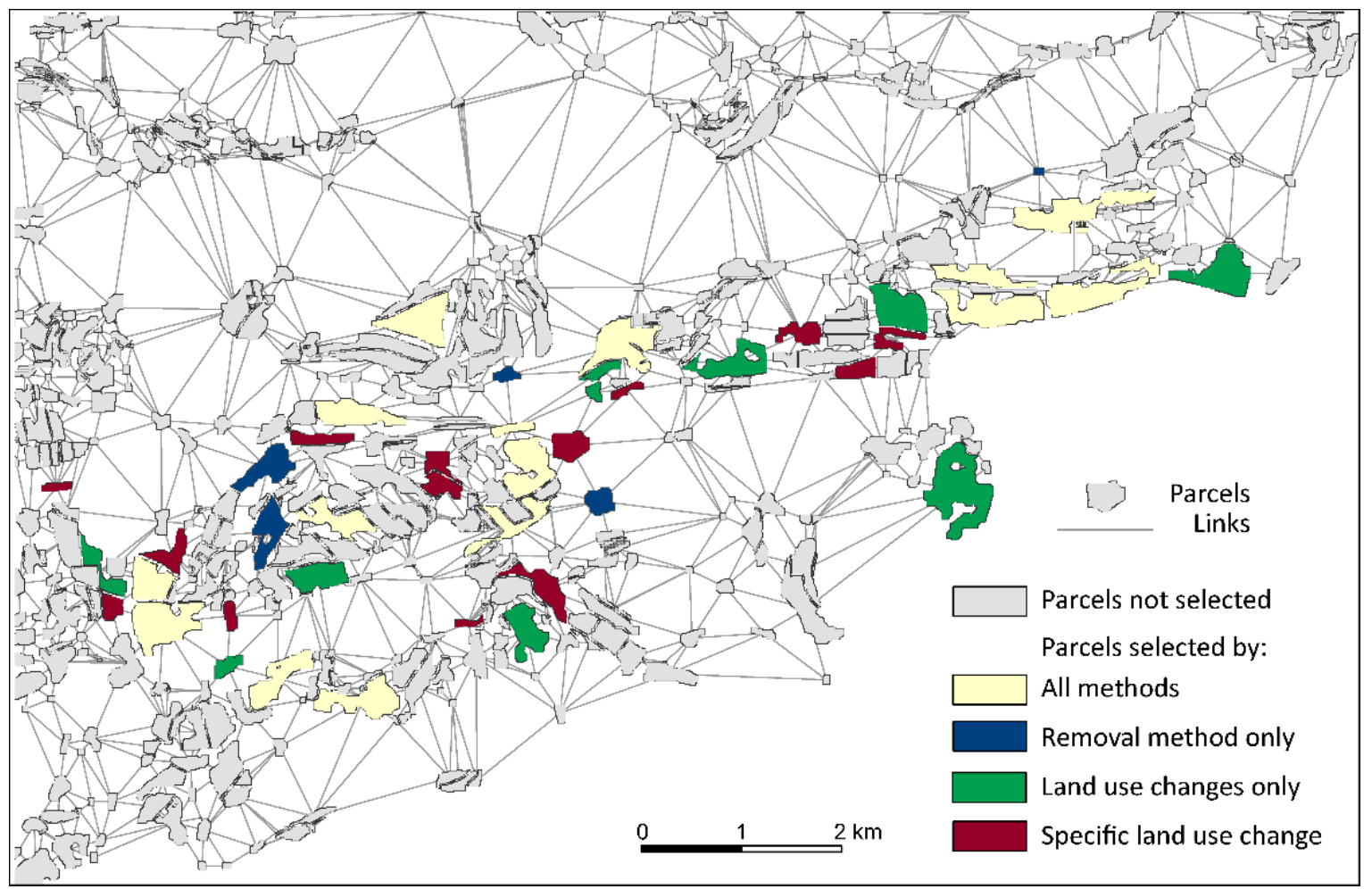

Figure 8 : Parcels selected among the 20 most important ones in terms of connectivity according to the removal method and the four land-use change scenarios. 


\section{Discussion}

The study presented focuses on the application of graph-based connectivity analyses to the local scale of agricultural parcels. Starting from the hypothesis that the node removal method applied to the parcel-based graph provides a reliable ranking compared to the application of land-use change scenarios, we have obtained a series of results worth discussing.

\subsection{Comparison of the connectivity values}

For the enumerative and cumulative approaches alike, the values obtained for parcel removal and land-use change indicate two types of behavior. On the one hand, parcel removal ( $P P C$ for the enumerative approach) invariably generates values representing a greater impact on connectivity, although the cereal crop scenario provides similar results. Except for $d P C_{\text {flux }}$, all the other metrics impact connectivity less. The separation into two groups can be interpreted as a function of the area involved each time a parcel is processed. As parcel removal is the most radical simulation, it was to be expected that this method would have the greatest impact. As the change from grassland to cropland affects the entire area of the parcels, it is also logical that this land-use change scenario should have a greater weight in absolute value.

It should be noted that all these comparisons might vary with the cost values assigned to the land-use categories, and to the spatial representation of specific actions such as the erection of perches. Here, perches were featured by $20 \mathrm{~m}$-radius discs to represent the area subjected to intense predation as observed in Andersson et al. (2009). But those authors report that this area depends both on the height of the perches and on visibility conditions. Moreover, the observed impact of predation follows a continuously decreasing curve from the perch up to a given distance away (e.g. $100 \mathrm{~m}$ ), that could be represented by a continuous variation in the cost values as in Foltête, Cosson \& Berthier (2008). But the same adjustment could be made for hedgerows and other sources of predation, which would result in a complex model in which interactions among parameters are not well controlled. Although our results could be enhanced by analyses of sensitivity to some of these parameters, they nonetheless confirm that logically the removal method largely overstates the impact of certain types of land-use changes when these changes concern only a part of the parcels.

\subsection{Does the removal method provide rankings similar to more realistic simulations?}

The main objective of the analyses was to compare the rankings determined by each method. The first results obtained by the enumerative approach applied to all the parcels confirmed the initial hypothesis, because all the rankings were very similar when considering Spearman correlation coefficients. More specifically, the main metric derived from the removal method $(d P C)$ yielded results that are highly correlated to those obtained under the cereal crop and perch erection scenarios. The correlation with the results under the hedgerow planting scenario was weaker, and in this case $d P C_{\text {connector yielded a more similar ranking than }} D P C$. At this step, one might question the utility of the parcel-based graph when a patch-based graph could be applied more easily because of the small number of nodes involved. For information purposes, $A P C$ from parcels and $D P C$ from patches were compared (by assigning to parcels the rank of the patch in which they are contained). The Spearman correlation coefficient was significant $(p<0.001)$ but much smaller $(0.22)$ than the coefficients linking all the other metrics, which were consistently higher than 0.8 . This confirms the specific character of the results from the parcel-based approach. 
As the inclusion of all the parcels in the analysis may mask potential differences, investigations were then conducted on the parcels selected in the leading ranks (top 5, top 10, and top 20), that are likely to be the most useful for decision support. In this case, comparing the parcels selected shows that the removal method produces variable results when taking the ranking yielded by the land-use change scenarios as a reference. Compared to the cereal crop and perch erection scenarios, the $d P C$ index generates the same top 20 (with only one difference) and a similar top 5 and top 10. Globally, these results are even better with $d P C_{\text {flux. }}$. On the other hand, the $d P C$ index fails to reproduce the hedgerow planting scenario (as illustrated in Fig. 4), and obtains quite poor results with the scenario combining hedgerows and perches. Once again, this variability can be interpreted as a dependence on the parcel area, because the simulated hedgerows are here planted along the parcel perimeters, and their impact on connectivity is probably less dependent on their areal extent and more closely related to their shape. Such an interpretation leads us to state that (1) the capacity of the $I P C$ index to produce a ranking similar to those obtained under the land-use change scenarios depends on the type of change and on the way this change is represented on the land-use map, and (2) this capacity seems to be greater if the land-use change is uniformly distributed among the parcels, giving more importance to larger parcels. This is supported by the fact that the behavior of $d P C_{\text {intra }}$ and $d P C_{\text {flux }}$ is similar to $d P C$, unlike $d P C_{\text {connector }}$ which is related more to land-use changes mainly affecting the links, as with the hedgerows in the present case.

The results of the cumulative approach show the same dependence of the similarity ranking on the type of land-use change. However, the difference between the four scenarios is less marked here. Most of the similarity values are moderate, meaning that the set of key parcels selected using the $d P C$ index has a common part with the sets defined under the land-use change scenarios, but never strictly corresponds to the results provided by any specific scenario. The fact that the removal method gives poorer results in the second part of the analysis can be explained by the chain effect induced by the cumulative approach in the selection of the "next best" parcels. In other words, following this stepwise procedure, the selection of a given parcel depends on the parcel selected at the previous step, and so influences the choice of the parcel selected next. This shows that when considering the cumulative effect of the elementary changes (i.e. the change affecting a given parcel) on connectivity, the removal method cannot exactly reproduce the ranking obtained from land-use changes, unlike our initial hypothesis.

\subsection{Contribution to the operational dimension of landscape graphs}

All these findings improve our knowledge of the operational dimension of landscape graphs. Several earlier studies have already investigated the sensitivity to certain criteria of the prioritization determined from connectivity metrics. But these studies focused on the way the removal method was applied at patch level, comparing for example patch ranking according to the type of distance (leastcost distances or resistance distances) for defining inter-patch links (Avon \& Bergès, 2016), or testing the sensitivity of connectivity metrics to the level of spatial aggregation in the definition of the patches (Blazquez-Cabrera et al., 2014). Those studies are significant contributions, because patches and links are the basic elements of any landscape graph. But in the case of agricultural landscapes, the transposition of connectivity analyses to operational issues also raises the question of the management units to be considered. As the most concrete actions that may be guided by a graph- 
based analysis involve effecting a change in agricultural practice and landscaping, the present analysis was to start from a more precise spatial scale using parcel-based graphs, and to contrast parcel removal with more realistic representations of field actions. It appears that such a method overstates connectivity levels because node removal corresponds to a stylized representation of reality.

\subsection{Two contexts for using parcel rankings as a guide for field action}

The results finally lead us to specify the relevant area of the removal method for carrying out field actions. Considering all the domains in which the use of landscape graphs is of interest, this leads us to distinguish two contexts for prioritization in the field. A first case would be the large capacity for action in many locations, where a massive operation is planned in the area under study. This assumes the availability of substantial financial support and the involvement of numerous actors (landowners, local authorities). In this case, a certain redundancy will occur among the numerous parcels involved, making the enumerative procedure suitable even though the cumulative procedure is likely to provide a best combination of parcels. Furthermore, the application of the cumulative approach for selecting many parcels (e.g., more than 100) is very time-consuming. The removal method can thus be considered a reliable approach, provided that the field actions affect the parcels totally (as with the crop scenario in the present study) or uniformly (as with perch scenarios). However, if very few landscape elements are to be potentially concerned by the actions in the field, their complementarity should be considered a prime criterion. This refers thus to the cumulative procedure based on a stepby-step selection of the key elements. In this case, our results suggest that the prioritization determined by the removal method is not consistent with the application of land-use change scenarios, because the chain effect of the step-by-step selection often makes the results differ. According to Fig. 6 , such differences could be minimized by outlining that large and connected parcels (or patches in other contexts) are usually selected among the key elements, whatever the selection method. However, users have to keep in mind that field actions that do not uniformly cover the parcels (such as hedgerows in the present case) cannot be properly prioritized by using the removal method. Further analyses would be needed to address the same question in different geographical contexts and to confirm the generalization of the outcomes.

\section{Conclusion}

In this paper, parcel-based graphs were constructed to identify the best locations at which to carry out field actions against the montane water vole, a grassland rodent. Several scenarios of landuse changes were simulated with the aim of ranking the parcels according to their effect on connectivity. Our hypothesis was that the node removal method applied to the parcels provides a ranking similar to those resulting from land-use change scenarios. As expected, the results have shown that the variation in connectivity induced by parcel removal was always overstated compared to the land-use change method. When prioritizing the parcels in an enumerative manner, the removal method provides similar rankings to land-use change scenarios, except for specific actions such as hedgerow planting. If the prioritization is done by cumulating the impact of each parcel using a stepby-step procedure, the removal method proves unreliable. Nonetheless, this last approach assumes that the field actions are restricted to very few cases, in places where actions must have complementary roles towards connectivity. 


\section{Acknowledgments}

This research was funded by the French Ministry of Ecology (Campagraphe project in the DIVA3 Program). It forms part of the Jurassian Arc long-term ecological research site (http://zaaj.univfcomte.fr/?lang=en). I wish to thank Antony Philibert and Damien Roy for their help in the mapping of the hedgerows.

Appendix 1. Resistance values used for computing least-cost distances.

\begin{tabular}{|l|r|}
\hline Land-use classes & \multicolumn{1}{|c|}{ Cost value } \\
\hline Buildings & 50 \\
\hline Crops & 25 \\
\hline Grasslands & 1 \\
\hline Other herbaceous areas & 1 \\
\hline Hedgerows & 25 \\
\hline Deciduous forests & 1000 \\
\hline Evergreen forests & 50 \\
\hline Mixed forests & 1000 \\
\hline Rivers & 1000 \\
\hline
\end{tabular}

\section{References}

Andersson, M., Wallander, J., \& Isaksson, D. (2009). Predator perches: a visual search perspective. Functional Ecology, 23, 373-379.

Avon, C., \& Bergès, L. (2016). Prioritization of habitat patches for landscape connectivity conservation differs between least-cost and resistance distances. Landscape Ecology, 31(7), 1551-1565.

Baguette, M., Blanchet, S., Legrand, D., Stevens, V.M., \& Turlure, C. (2013). Individual dispersal, landscape connectivity and ecological networks. Biological Reviews, 88, 310-326.

Berthier, K., Piry, S., Cosson, J.F., Giraudoux, P., Foltête, J.C., Defaut, R., Truchetet, D., \& Lambin, X. (2014). Dispersal, landscape and travelling waves in cyclic vole populations. Ecology Letters, 17, 53-64.

Blant, M.A., Beuret, B., Poitry, R., \& Joseph, E. (2009). Influence of landscape and soil on the intensity of pullulations of vole (Arvicola terrestris scherman) in Swiss Jura. Revue Suisse Agriculture, 41(5), 301-307.

Blazquez-Cabrera, S., Bodin, O., \& Saura, S. (2014). Indicators of the impacts of habitat loss on connectivity and related conservation priorities: Do they change when habitat patches are defined at different scales? Ecological Indicators, 45, 704-716.

Bonnet, T., Crespin, L., Pinot, A., Bruneteau, L., Bretagnolle, V., \& Gauffre, B. (2013). How the common vole copes with modern farming: Insights from a capture-mark-recapture experiment. Agriculture, Ecosystems and Environment, 177, 21-27.

Calabrese, J.M., \& Fagan, W.F. (2004). A comparison-shopper's guide to connectivity metrics. Frontiers in Ecology and the Environment, 2(10), 529-536. 
Clauzel, C., Bannwarth, C. \& Foltête, J.C. (2015). A planning tool for integrating broad-scale connectivity in habitat restoration: an application to pond creation in eastern France. Journal of Nature Conservation, 23, 98-107.

Coeurdassier, M., Riols, R., Decors, A., Mionnet, A., David, F., Quintaine, T., Truchetet, D., Scheifler, R., \& Giraudoux, P. (2014). Unintentional wildlife poisoning and proposals for sustainable management of rodents. Conservation Biology, 28, 315-321.

Delattre, P., \& Giraudoux, P. (eds.) (2009). Le campagnol terrestre : prévention et contrôle des populations. QUAE Edition, Versailles.

Duhamel, R., Quéré, J.P., Delattre, P., \& Giraudoux, P. (2000). Landscape effects on the population dynamics of the fossorial form of the water vole (Arvicola terrestris sherman). Landscape Ecology, 15, 89-98.

Fall, A., Fortin, M.J., Manseau, M., \& O’Brien, D. (2007). Spatial graphs: principles and applications for habitat connectivity. Ecosystems, 10, 448-461.

Foltête, J.C., Clauzel, C., \& Vuidel, G. (2012). A software tool dedicated to the modelling of landscape networks. Environmental Modelling \& Software, 38, 316-327.

Foltête, J.C., Cosson, J.F., \& Berthier, K. (2008). Cost-distance defined by a topological function of landscape. Ecological Modelling, 210, 104-114.

Foltête, J.C., Couval, G., Fontanier, M., Vuidel, G., \& Giraudoux, P. (2016). A graph-based approach to defend agro-ecological systems against water vole outbreaks. Ecological Indicators, 71, 87-98.

Foltête, J.C., Girardet, X., \& Clauzel, C. (2014). A methodological framework for the use of landscape graphs in land-use planning. Landscape and Urban Planning, 124, 140-150.

Foltête, J.C., \& Giraudoux, P. (2012). A graph-based approach to investigating the influence of the landscape on population spread processes. Ecological Indicators, 18, 684-692.

Foltête, J.C., \& Vuidel, G. (2017). Using landscape graphs to delineate ecologically functional areas. Landscape Ecology, 32, 249-263.

García-Feced, C., Saura, S., \& Elena-Rosselló, R. (2011). Improving landscape connectivity in forest districts: A two-stage process for prioritizing agricultural patches for reforestation. Forest Ecology and Management, 261, 154-161.

Galpern, P., Manseau, M., \& Fall, A. (2011). Patch-based graphs of landscape connectivity: A guide to construction, analysis and application for conservation. Biological Conservation, 144, 44-55.

Girardet, X., Foltête J.C., Clauzel C., \& Vuidel G. (2016). Restauration de la connectivité écologique : proposition méthodologique pour une localisation optimisée des passages à faune. Vertigo URL: http://vertigo.revues.org/17337

Gurrutxaga, M., \& Saura, S. (2013). Prioritizing highway defragmentation locations for restoring landscape connectivity. Environmental Conservation, 41, 157-164.

Jacob, J., \& Hempel, N. (2003). Effects of farming practices on spatial behaviour of common voles. Journal of Ethology, 21, 45-50.

Jug, D., Brmez, M., Ivezic, M., Stipesevic, B., \& Stosic, M. (2008). Effect of different tillage systems on populations of common voles (Microtus arvalis Pallas, 1778). Cereal Research Communications, 36, 923-926.

Kay, B.J., Twigg, L.E., Nicol, H.I., \& Korn, T.J. (1994). The use of artificial perches to increase predation on house mice (Mus domesticus) by raptors. Wildlife Research, 21(1), 95-106.

Keitt, T.H., Urban, D.L., \& Milne, B.T. (1997). Detecting critical scales in fragmented landscapes. Conservation Ecology, 1(1), 4. URL: http://www.consecol.org/vol1/iss1/art4/

Loro, M., Ortega, E., Arce, R.M., \& Geneletti, D. (2015). Ecological connectivity analysis to reduce the barrier effect of roads. An innovative graph-theory approach to define wildlife corridors with multiple paths and without bottlenecks. Landscape and Urban Planning, 139, 149-162.

Michel, N., Burel, F., \& Butet A. (2006). How does landscape use influence small mammal diversity, abundance and biomass in hedgerow networks of farming landscapes? Acta Oecologia, 30, 11-20.

Mimet, A., Clauzel, C., \& Foltête, J.C. (2016). Locating wildlife crossings for multispecies connectivity across linear infrastructures. Landscape Ecology, 31, 1955-1973.

Morilhat, C., Bernard, N., Foltête, J.C., \& Giraudoux, P. (2008). Neighbourhood landscape effect on population kinetics of the fossorial water vole (Arvicola terrestris scherman), Landscape Ecology, 23, 569-579. 
Ott, R.L., \& Longnecker, M. (2015). An Introduction to Statistical Methods and Data Analysis. Cengage Learning Custom Publishing, Boston.

Pascual-Hortal, L., \& Saura, S. (2006). Comparison and development of new graph-based landscape connectivity indices: towards the prioritization of habitat patches and corridors for conservation Landscape Ecology, 21, 959967.

Sahraoui, Y., Foltête, J.C., \& Clauzel, C. (2017). A multi-species approach for assessing the impact of land-cover changes on landscape connectivity. Landscape Ecology, 32, 1819-1835.

Saura, S., \& Pascual-Hortal, L. (2007). A new habitat availability index to integrate connectivity in landscape conservation planning: Comparison with existing indices and application to a case study. Landscape and Urban Planning, 83, 91-103.

Saura, S., \& Rubio, L. (2010). A common currency for the different ways in which patches and links can contribute to habitat availability and connectivity in the landscape. Ecography, 33, 523-537.

Taylor, P., Fahrig, L., \& With, W. (2006). Landscape connectivity: A return to basics. In K. R. Crooks \& M. Sanjayan (Eds.), Connectivity Conservation (pp. 29-43). Cambridge, Cambridge University Press.

Urban, D.L., Minor, E.S., Treml, E.A., \& Schick, R.S. (2009). Graph models of land mosaics. Ecology Letters, 12, 260-273.

Vogt, P., Riiters, K.H., Iwanowski, M., Estreguil, C., Kozak, J., Wade, T.G., \& Wickham, J.D. (2007). Mapping spatial patterns with morphological image processing. Landscape Ecology, 22, 171-177.

Zetterberg, A., Mörtberg, U.M., \& Balfors, B. (2010). Making graph theory operational for landscape ecological assessments, planning, and design. Landscape and Urban Planning, 95, 181-191. 